\title{
Prospective evaluation of a nonsurgical device for rhinoplasty*
}

Marcel A. Deggeller, David Holzmann, Michael B. Soyka

Department of Otorhinolaryngology, Head and Neck Surgery, University Hospital Zurich, Zurich, Switzerland
Rhinology 56: 73-81, 2018

https://doi.org/10.4193/Rhino17.133

*Received for publication:

June 19, 2017

Accepted: October 10, 2017

Background: Rhinoplasty represents one of the most challenging and frequently performed procedures in plastic surgery and non-surgical rhinoplasty is rarely considered. The aim of this study was to investigate whether the "Nasella Nose Former" (NNF), a newly developed non-surgical rhinoplasty device, could improve objective and subjective results following surgical rhinoplasty and even correct the shape of the nose without any surgery at all.

Methodology: In this prospective, monocentric, two-armed, non-blinded randomized, controlled clinical trial, a total of 43 participants were included. In the Surgical group, 22 patients undergoing open or closed rhinoplasty with osteotomies were randomised based on their birth year; 15 of them got to wear the NNF over 8 weeks postoperatively and 7 patients getting surgery without the NNF formed the control group. In the Cosmetic group, 21 participants wore the NNF without surgery over 14 months. At every follow-up exam, angles for crookedness, nasal hump and width were measured, the investigator assessed the patient's nose and asked for patient satisfaction using a Likert-scale.

Results: Patients in the Surgical group wearing the NNF did not show any significant difference concerning objective measurements, investigator assessments and patient satisfaction compared to those not wearing the NNF. In the Cosmetic group, participants did not show objective improvements in measurements and investigator assessment. However, participants were significantly more satisfied after 14 months with their nasal back, nasal axis and outer nose in general.

Conclusions: Considering the results of this study, we conclude that this perfectly customised external device to enhance surgical rhinoplasty outcomes or correct the shape of the nose without surgery does not seem to be effective and that further investigations in this field are not meaningful.

Key words: rhinoplasty, nonsurgical device, nose deformities, nasal bone, cosmetic techniques

\section{Introduction}

Across different cultures and periods of time, there has always been a human want to fit certain body standards. Especially the shape of the nose seems to be very important for human self-perception ${ }^{(1)}$. That is why since ancient times, surgeons have tried to improve the appearance of a patient's nose. It is therefore of no surprise that according to statistics released by the American Society of Plastic Surgeons in 2016, rhinoplasty is ranked on third place amongst the most requested fields of plastic surgery ${ }^{(2)}$.
The idea to influence the shape of the nose without surgery is not new either. One established nonsurgical method to alter the shape of the nose is the use of nasal injections with dermal fillers such as hyaluronic acid, calcium hydroxylapatite gel or silicone. Many rhinoplasty surgeons have used fillers in the nose for years both as an alternative to surgery as well as to correct deformities following cosmetic rhinoplasty ${ }^{(3-5)}$. Besides that, there is a variety of nasal devices on the market that claim to offer easy mechanical solutions to reshape the nose, especially in Asia. However, there has not been any scientific evidence for the 
efficacy of any of those mechanical devices yet.

In this study, we investigated a new customised device for external use, named the "Nasella Nose Former" (NNF). The theoretical fundament of the NNF is based on the theory that applying ongoing physical stress and strain on bone can lead to bone reorganization and reshaping ${ }^{(6,7)}$. The NNF is mainly inspired by dental braces in orthodontia where constant tension and stress applied to the teeth and/or the jaw can lead to correction of both tooth position as well as jaw shape by permanent bone remodelling ${ }^{(8-10)}$. The inventors hypothesised that the NNF could induce bone reorganisation by applying pressure on the nasal bone and frontal bone of the maxilla. On the one hand, this could be used to stabilize the nose after nasal surgery to prevent secondary distortion and equalizing deformities caused by soft-tissue forces, thus leading to better patient outcomes and satisfaction. On the other hand, the NNF is thought to be an alternative to nasal surgery in the first place. The inventors claimed that the NNF could be used to correct different nose deformities such as crooked nose, nasal hump and wide nose. Until this day, no clinical trials were performed, but pre-clinical tests by the developers showed promising results.

The aim of this study was to investigate whether the NNF could improve objective and subjective results following surgical rhinoplasty and even correct the shape of the nose without any surgery at all.

\section{Materials and methods}

\section{Study design and participants}

The study was designed as a prospective, monocentric, two-armed, non-blinded randomized, controlled clinical trial carried out by the ear, nose and throat (ENT) department of the University Hospital of Zurich and was executed from January 2014 to March 2017. This study was approved by the cantonal ethics committee of Zurich. Informed consent was obtained from all subjects prior to any intervention. The participants were divided into two major groups; in the following called "Surgical group" and "Cosmetic group". On the one hand, the first group contained patients who received nasal surgery prior to the use of the NNF ("Surgical group with Nasella"). On the other hand, patients who got nasal surgery but did not get to wear the NNF formed the corresponding control group ("Surgical group without Nasella"). All open or closed rhinoplasties with osteotomies within the "Surgical group" were performed by the senior author at the University Hospital of Zurich. The second group included those patients who agreed to wear the NNF in the first place without surgery. As there is no comparable product on the market, there was no reference for the Cosmetic group.

Patients presenting at the ENT-department of the University Hospital of Zurich for surgical rhinoplasties were informed about a possible postoperative use of the NNF. In case of an agreement, these patients were assigned to the "Surgical group" and randomized based on their birth year. Odd-numbered years were assigned to the "Surgical group with Nasella" and evennumbered to the "Surgical group without Nasella". For the "Cosmetic group", patients with no wish for surgery were recruited from the ENT-clinic as well as by an advertisement.

As the NNF was developed to treat bony deformities, patients with crooked nose and/or nasal hump and/or wide nose were included. Patients had to be aged 18 years or older and in good health condition, they had to be fully informed and a consent form had to be signed. Exclusion criteria were known or suspected non-compliance, drug or alcohol abuse, inability to follow the research plan (e.g. due to language barrier, psychiatric disorder or dementia), allergy to any component of the NNF, legally incapable patients, known metabolic disease of the bones and the use of drugs that influence bone metabolism.

\section{Nasella Nose Former (NNF)}

The development of the NNF started in 2004 and in 2012 a European patent was granted. Following the Medical Device Directive (Council Directive 93/42/EEC of 14 June 1993 concerning medical devices, OJ No L 169/1 of 1993-07-12), the NNF is classified as a non-invasive device Class 1.

From each participant's nose, an individual mould was taken using the casting compound Epiform-solid (Dreve Dentamid $\mathrm{GmbH}$, Germany). With the mould, a plaster model was grouted which was used to fabricate an individual mask with deepdrawing method using transparent Erkoloc-pro deep-drawing foil (ERKODENT Erich Kopp GmbH, Germany). Parts of the mask corresponding to areas where pressure on the nose was desired were padded with COMPEED blister plasters (Johnson \& Johnson AG, Switzerland). The mask was then connected to an elastic headband via a metal clasp and a security module (Figure 1). In the production of the NNF, only certified and well-tried material from orthodontia was used.

\section{Photo-documentation \& measurements}

Photo-documentation and measurements were performed with the archiving and visualization software "synedra View 16" of the University Hospital of Zurich (Version 16.0.0.6, synedra information technologies $\mathrm{GmbH}$, Austria). Standardised pictures were taken according to Strub et al. ${ }^{(11)}$.

To measure crooked nose deformities, the angle between a line from the glabella at the top level of the eye brows to the middle of the philtrum and a line from the glabella at the top level of the eye brows to the middle of the bony nasal dorsum was measured in degrees on a straight frontal photograph. To objectify nasal hump deformities the angle between the line from the nasion to the tip and the line from the nasion to the maximal bony hump deformity was measured in degrees on a side view profile photograph. For wide nose deformities, the relative ratio between the distance between the left to the right canthus and 


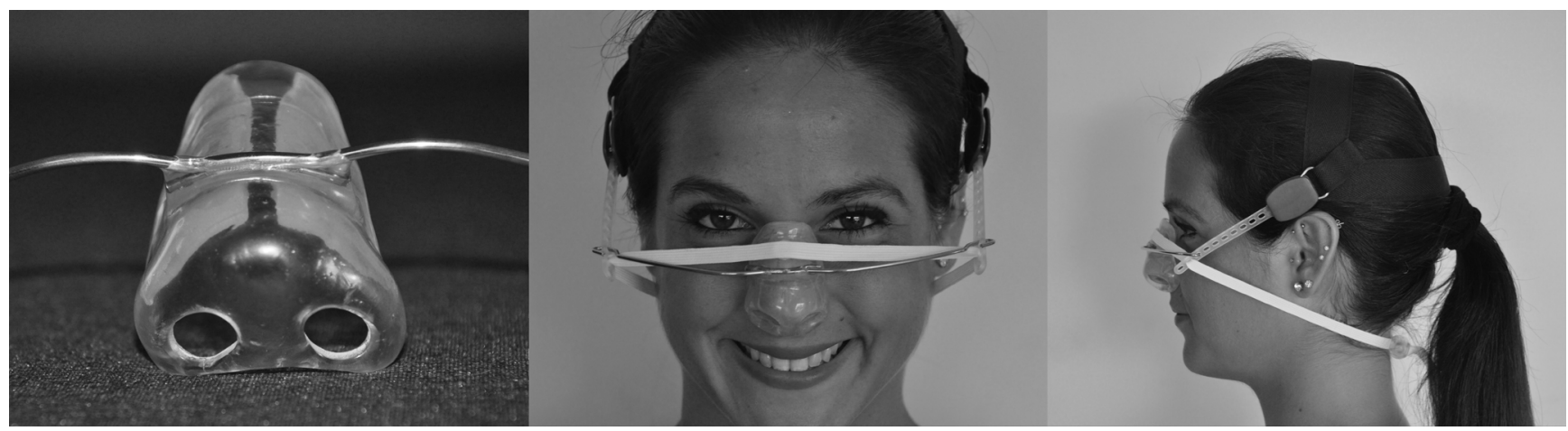

Figure 1. Nasella Nose Former. Individually adjusted nasal mask padded with blister plasters connected to an elastic headband via a metal clasp and a security module.
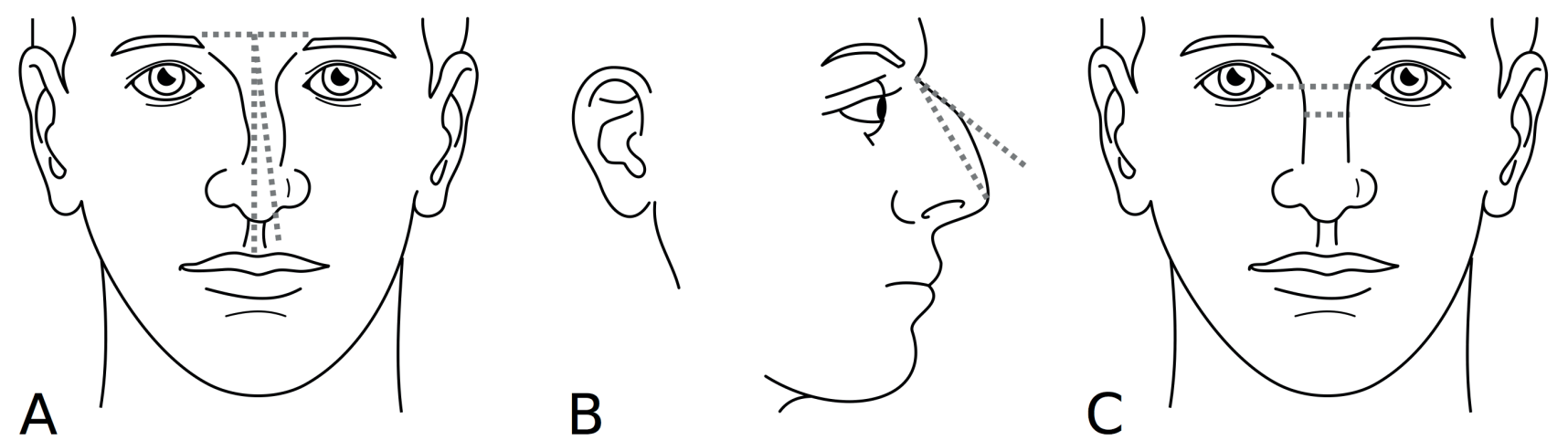

Figure 2. Schematic representation of the measured angles for crooked nose deformities (A), nasal hump (B) as well as the proportion for wide nose deformities (C).

the width at the broadest part of the bony nasal dorsum on a straight frontal photograph was calculated (Figure 2).

\section{Screening}

An overview of the course of the study is presented in Figure 3. At the first presentation, the investigator collected demographic data such as date of birth, age at informed consent and gender from all participants as well as concurrent diseases and medication. Furthermore, inclusion and exclusion criteria were checked. The patient's nose was assessed for crookedness, nasal hump and width. Finally, the investigator divided patients into their corresponding groups.

\section{Surgical group with Nasella}

Immediately after open or closed rhinoplasty with osteotomies was performed, still under anaesthesia, a mould of the patient's nose was taken and the patient received a thermoplastic nasal cast. After seven days, the cast was removed and the NNF was adjusted. This visit corresponds to "Nasella Start" in Figure 3. The investigator assessed the patient's nose for crookedness, degree of nasal hump, visible edges on the nasal back and width of the nose, each on a Likert-scale from 1 (no pathology) to 4 (major pathology). For documentation and measurements, pictures from the front, left and right side were taken.

From this point on, the patient had to wear the NNF during eight weeks corresponding to the following scheme:

$$
\begin{array}{lll}
\text { - } & \text { 1. week: } & \text { 3x a day for 15-30 minutes } \\
\text { - } & \text { 3. and 4. week: } & 3 x \text { a day for 15-30 minutes } \\
\text { - } & \text { 5. to 8. week: } & \text { 2x a day for 30 minutes } \\
\end{array}
$$

After a period of one, two and four weeks, follow-up examinations took place. Each follow-up examination lasted around 20 minutes. The investigator assessed the patient's nose as described above. Aberrations from the protocol were noted if the patient had not worn the device adequately. Any adverse event was documented. Furthermore, patients answered questions about satisfaction regarding the nasal hump, crookedness of the nose, the outer nose in general, nasal breathing and the product Nasella, each on a Likert-scale from 1 (very dissatisfied) to 5 (very satisfied). For documentation and measurements, pictures from the front, left and right side were taken. After eight weeks, the final examination was conducted following the same evaluation.

\section{Surgical group without Nasella}

This group followed the same procedure as the "Surgical group with Nasella" except that these patients did not get to wear the 

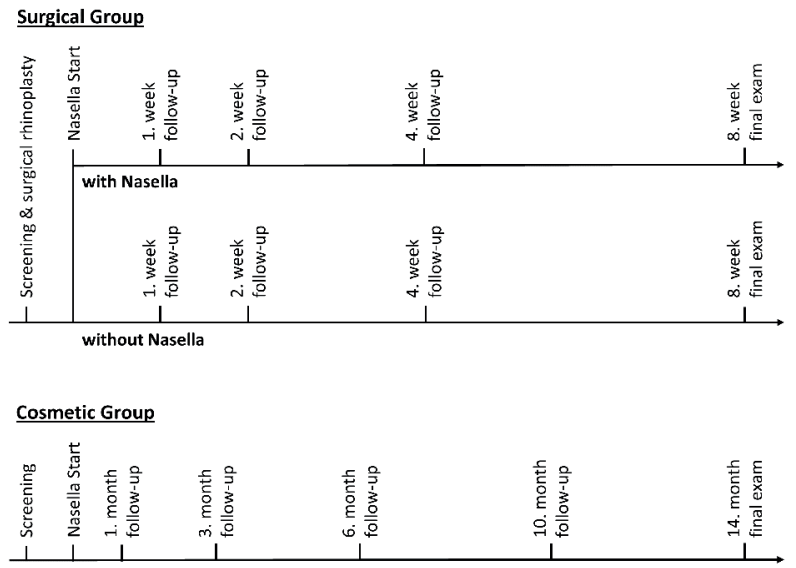

Figure 3. Overview of the course of the study.

NNF after the removal of the thermoplastic cast. The use of a placebo-device would have been meaningless since patients would have noticed the lack of pressure.

\section{Cosmetic group}

Patients in this group did not undergo nasal surgery. Instead, a mould as well as pictures of the patient's nose were taken in the first place. After approximately seven days, the NNF was individually adjusted and the same procedure was conducted as for the "Surgical group with Nasella".

From this point on, the patient had to wear the NNF during 14 months corresponding to the following scheme:

- 1. month: $2 x$ a day for 10-20 minutes

- 2. month: $2 x$ a day for 20-30 minutes

- 3. month: $2 x$ a day for 30 minutes

- 4. to 14 . month: $1 x$ or $2 x$ a day for 30 or 20 minutes, respectively

After a period of one, three, six and ten months, follow-up examinations and a final examination after 14 months took place. Procedures and durations of these examinations were performed analogous to the "Surgical group with Nasella".

\section{Statistical analysis}

To determine the sample size, a power analysis with an assumed power of $95 \%$ and a level of significance of 0.01 was performed. Under the assumption that an improvement of $1^{\circ} \pm$ a SD of $0.41^{\circ}$ can be considered a good result, a minimal sample size of 6 participants per group was calculated. To compensate an eventual error of $30 \%$, we aimed for a total of 10 participants for both the "Surgical group with Nasella" and the "Surgical group without Nasella" and 20 participants for the "Cosmetic group". As the measured data showed a normal distribution, an independent samples t-test was used to analyse differences within the Surgical group ("with Nasella" versus "without Nasella") and a paired samples t-test within the Cosmetic group, respectively.
A
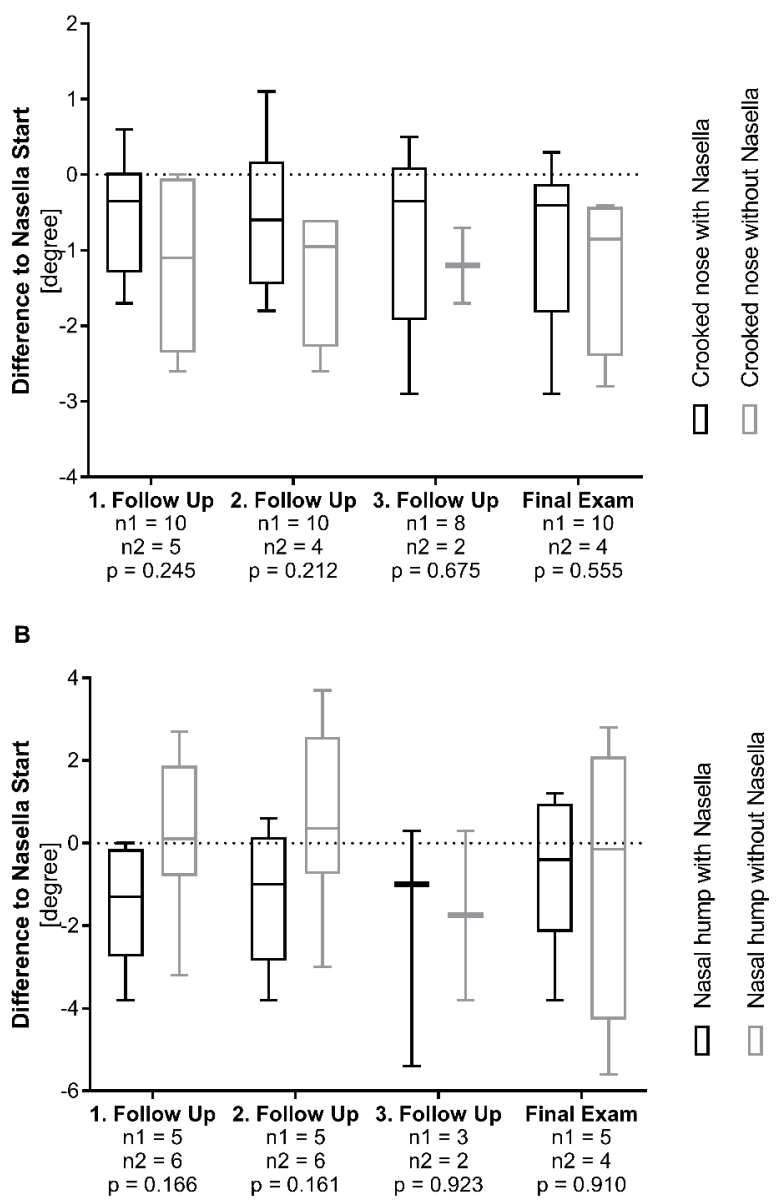

Figure 4. Objectively measured differences to Nasella Start in the Surgical group in degrees. A: Difference in angles for crooked nose deformities to Nasella Start. No significant difference was found between patients wearing the NNF compared to controls. B: Nasal hump deformities showed a slight but not significant tendency to smaller angles with the NNF at the first two follow-ups that disappears towards the final exam.

Patient satisfaction and investigator assessments were compared using the Mann-Whitney $U$ test for the Surgical group and the Wilcoxon test for the Cosmetic group respectively. In correlation analyses, the Spearman's rho test was used. The significance level alpha was set to 0.05 .

\section{Results}

The original population in this study consisted of 43 participants with an average age of 30 years (range 18 to 60 years). The remaining categorical data are summarized in Table 1 and Table 2.

Surgical group

Participants within the Surgical group did not show any demo- 
Table 1. Categorical data of the Surgical Group $(N=22)$.

\begin{tabular}{|c|c|c|c|c|c|c|}
\hline \multirow[t]{2}{*}{ Variable } & \multicolumn{3}{|c|}{ Surgical Group with Nasella } & \multicolumn{3}{|c|}{ Surgical Group without Nasella } \\
\hline & Frequency & Percent & $\mathbf{N}$ & Frequency & Percent & $\mathbf{N}$ \\
\hline Sex & & & 15 & & & 7 \\
\hline Female & 6 & 40 & & 4 & 57 & \\
\hline Male & 9 & 60 & & 3 & 43 & \\
\hline Crooked Nose & & & 15 & & & \\
\hline No & 3 & 20 & & 2 & 29 & 7 \\
\hline Yes & 12 & 80 & & 5 & 71 & \\
\hline Nasal Hump & & & 15 & & & 7 \\
\hline No & 8 & 53 & & 1 & 14 & \\
\hline Yes & 7 & 47 & & 6 & 86 & \\
\hline Wide Nose & & & 15 & & & 7 \\
\hline No & 13 & 87 & & 6 & 86 & \\
\hline Yes & 2 & 13 & & 1 & 14 & \\
\hline Rhinoplasty & & & 15 & & & 7 \\
\hline open & 2 & 13 & & 2 & 29 & \\
\hline closed & 13 & 87 & & 5 & 71 & \\
\hline Osteotomies & & & 15 & & & 7 \\
\hline No & 0 & 0 & & 0 & 0 & \\
\hline Yes & 15 & 100 & & 7 & 100 & \\
\hline $\begin{array}{l}\text { Nasal hump } \\
\text { reduction }\end{array}$ & & & 15 & & & 7 \\
\hline No & 7 & 47 & & 2 & 29 & \\
\hline Yes & 8 & 53 & & 5 & 71 & 7 \\
\hline
\end{tabular}

graphic differences between the two subgroups "with Nasella" and "without Nasella". There were no significant differences between patients wearing the NNF postoperatively and controls not wearing the NNF regarding angles for crooked nose and nasal hump at any point throughout the course of the study. However, patients with a nasal hump showed a slight tendency to smaller angles wearing the NNF after one and two weeks (Figure 4). The case number for overly wide noses was too small to perform any statistical test. Likewise, patient satisfaction regarding the nasal hump, crookedness, outer nose in general and nasal breathing did not differ significantly between the two groups (Figure 5). Investigator assessments for crookedness, nasal hump, edges of the nasal back and width of the nose did not show any significant differences between the two groups. From the initial 22 participants in the Surgical group, 18 finished the entire study. Three were lost to follow-up (two after the second, one after the third follow-up) and one dropped out due to lack of effectiveness after the second follow-up.

\section{Cosmetic group}

Interestingly, in the Cosmetic group, patients with a nasal hump showed significant bigger angles after one (paired samples t-test, $n=19, M=-1.605, S E=0.598, p=0.015)$, three $(n=15$, $M=-1.327, S E=0.455, p=0.011)$ and 14 months $(n=7, M=$ $-1.557, \mathrm{SE}=1.682, \mathrm{p}=0.05)$ compared to the first visit. However, no significant difference was found after six and ten months as well as for the intervals between each of the follow-up examinations. If we compare the first to the last measurement (after at least six months of follow-up), there is no significant difference neither $(n=15, M=-0.700, S E=0.475, p=0.163)$. Results for crooked and wide noses showed no differences at all (Figure 6). Concerning patient satisfaction, participants were more satisfied after 14 months with their nasal hump (Wilcoxon test, $n=7, p$ $=0.038)$, nasal axis $(p=0.026)$ and outer nose in general $(p=$ 
Table 2. Categorical data of the Cosmetic Group $(\mathrm{N}=21)$.

\begin{tabular}{|c|c|c|c|}
\hline \multirow[t]{2}{*}{ Variable } & \multicolumn{3}{|c|}{ Cosmetic Group } \\
\hline & Frequency & Percent & $\mathbf{N}$ \\
\hline Sex & & & 21 \\
\hline Female & 15 & 71 & \\
\hline Male & 6 & 29 & \\
\hline Crooked Nose & & & 21 \\
\hline No & 15 & 71 & \\
\hline Yes & 6 & 29 & \\
\hline Nasal Hump & & & 21 \\
\hline No & 0 & 0 & \\
\hline Yes & 21 & 100 & \\
\hline Wide Nose & & & 21 \\
\hline No & 14 & 67 & \\
\hline Yes & 7 & 33 & \\
\hline
\end{tabular}

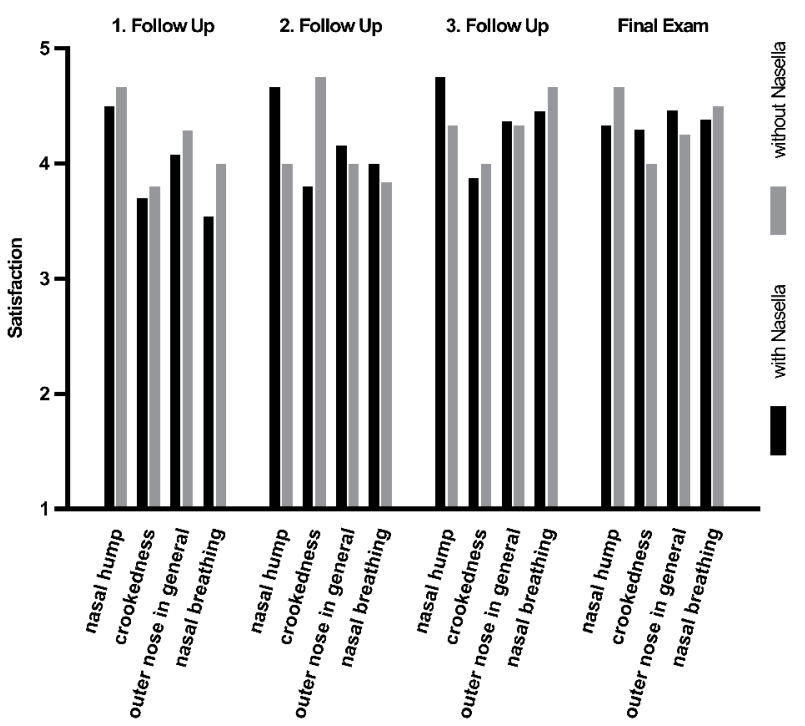

Figure 5. Subjective patient satisfaction in the Surgical group on a Likertscale from 1 to 5 . No significant differences between patients wearing the NNF and controls were found for nasal hump, crookedness, outer nose in general and nasal breathing at any point throughout the study.
0.023). No significant difference for satisfaction was found for nasal breathing and product Nasella (Figure 7). However, the rating for product Nasella showed significant lower satisfaction at the final examination compared to the second to last visit (Wilcoxon test, $\mathrm{n}=7, \mathrm{p}=0.038$ ). A Spearman's rho test did not show any correlation between the objective measurements and the subjective patient satisfaction for patients with nasal hump. Investigator assessments for crookedness, nasal hump, edges of the nasal back and width of the nose did not show any significant differences. From the initial 21 participants in the Cosmetic group, only 7 ran through the entire course of the study. One participant withdrew right after the initial visit, two after the first, three after the second, six after the third and two after the fourth follow-up. Reasons for drop-outs were "lost to follow-up" $(n=8)$, "protocol violation" ( $n=7)$, "lack of effectiveness" $(n=5)$, "adverse event" $(n=1)$, and "withdrawal of consent" $(n=1)$.

\section{Adverse events / severe adverse events}

Reported adverse events related to the use of the NNF included two cases of headache, one case of local pressure and pain, two cases of visible reddened skin, one case of visible pressure mark and two cases where the NNF impacted on a wrong part of the nose. Only one of the latter led to early termination of the participation. All others continued the course of the study. There were no severe adverse events.

\section{Discussion}

In this study, we investigated whether the Nasella Nose Former (NNF) could improve outcomes and increase patient satisfaction after rhinoplasty. Furthermore, the effectiveness of the NNF as an alternative to nasal surgery was tested. We did not observe any positive postoperative effects neither in the objective rhinoplasty results nor in the investigator's assessments nor in patient satisfaction. Interestingly, patients were significantly more satisfied with their noses after a cosmetic use of the NNF without surgery even though the analyses did neither show better objective outcome measurements nor higher scores in the investigator's assessments.

The results of this study imply that an external device like the NNF - at least in the present setting and type of application - is not an effective option to support or replace surgical rhinoplasty. Especially, since a medical device should provide positive outcomes in most cases to qualify for sales and distribution. The inventors of the NNF were mainly inspired by dental braces in orthodontia. One reason why the NNF was not successful might be that it was not worn permanently in contrast to the latter. The NNF might have been more successful with more continuous periods of application. Another important point to consider when talking about tooth movement is that teeth are not fixed in the jaw but connected to the alveolar bone via the periodontal ligament (PDL). It is known that the PDL plays a crucial role in orthodontic tooth movement ${ }^{(9,12-14)}$. Therefore, orthodontic mechanisms can probably not be applied to the nose without limitations.

We suspect that patients in the Cosmetic group reported higher satisfaction after the use of the NNF due to a certain placebo effect as participants had to wear the medical device over a long period of time and received a lot of medical attention. It 
A

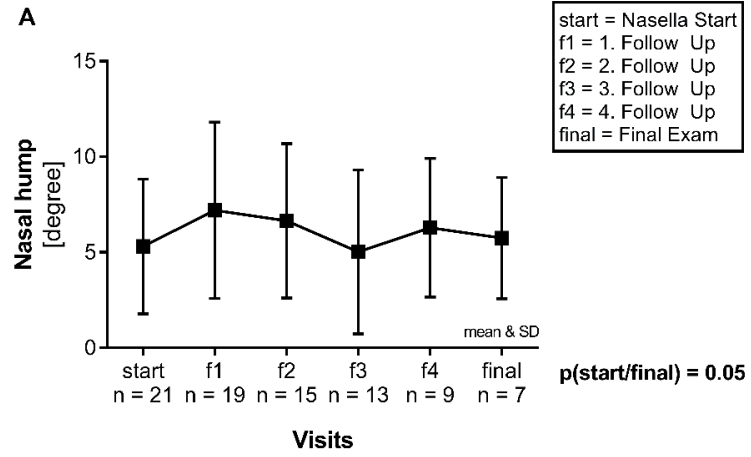

B

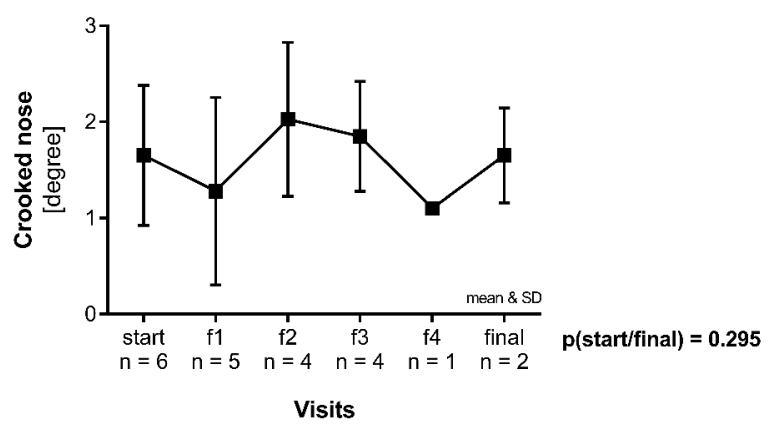

C

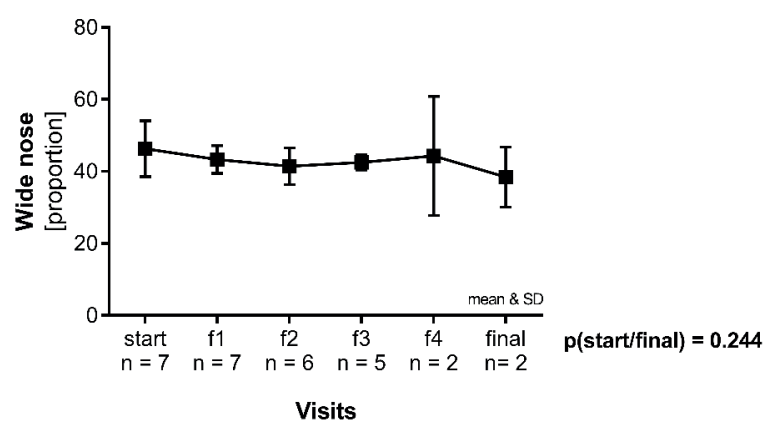

Figure 6. Objective measurements in the Cosmetic group. Participants with nasal hump deformities showed significant larger angles after one, three and 14 months but not after six and ten months (A). No significant differences were found for crooked nose (B) and wide nose deformities (C).

is known that placebo effects primarily address subjective and self-appraised symptoms and that the effect is dramatically enhanced by clinician interactions ${ }^{(15,16)}$. Furthermore, it is generally accepted that the extent of a placebo response is related to the degree of invasiveness of the procedure. The use of injections for example leads to a higher placebo response than oral application. The same is true for sham devices and surgical procedures $(17,18)$. The effect is even stronger if the expected outcome is desired by the participant. Greenwald et al. called this phenomenon illusory placebo effect ${ }^{(19)}$. This could explain why some

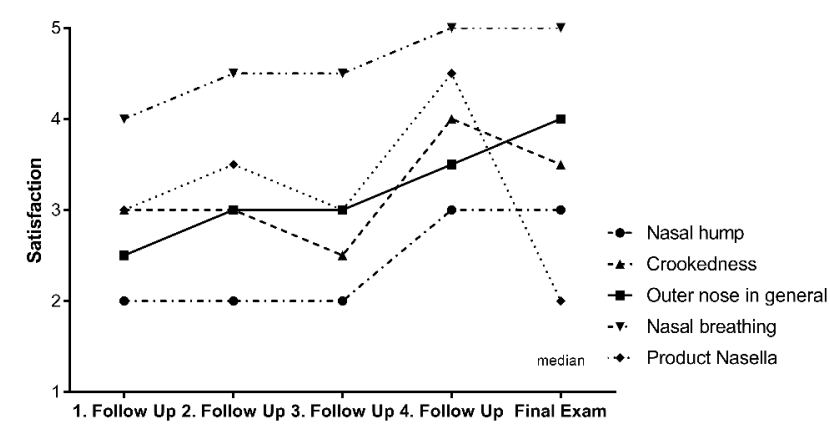

Figure 7. Subjective patient satisfaction in the Cosmetic group on a Likert-scale from 1 to 5. Participants showed significant improvement in satisfaction for nasal hump, crookedness and outer nose in general from the first follow-up to the final exam. For the rating of the product Nasella, a significant drop in satisfaction from the fourth follow-up to the final exam occurred.

participants in this study were more satisfied with their noses after wearing the NNF although no objective improvement was observed. Another explanation might be that participants who did not experience a subjective improvement dropped out of the study over time thus leaving mostly satisfied participants behind. Furthermore, the performed measurements might not be sensitive enough to small changes, only noticeable by the patient, due to limited accuracy.

Within the Cosmetic group, patients showed a significant drop of satisfaction regarding the product Nasella from the fourth follow-up to the final examination although satisfaction levels for nasal hump, crookedness and outer nose in general raised. A possible explanation could be that patients were more and more satisfied with their noses during the study but did not reach the desired result in the end which might have led to an overall disappointment regarding the product.

A common consequence of rhinoplasty is postoperative oedema and swelling which can mask the final rhinoplasty result for weeks or even months after surgery. In fact, postoperative oedema after rhinoplasty decreases by two-thirds within the first month and by 95 percent within six months ${ }^{(20)}$. Ozucer et al. presented evidence that post-rhinoplasty taping decreases postoperative swelling by compressing the skin envelope to the underlying cartilaginous framework ${ }^{(21)}$. It is possible that the NNF has a similar effect which could explain why, within the Surgical group, patients with nasal hump wearing the NNF showed a tendency to better outcomes after one and two weeks compared to the Surgical group without the NNF. However, after eight weeks, this difference is no longer visible, probably due to natural resolution of swelling in the control group.

Participants in the Cosmetic group showed significant worse angles for nasal hump after one, three and 14 months, but not after six and ten months and neither for the intervals between 
each visit. This could be by chance since repeated testing occasionally leads to significant results. Another possibility is that the use of the NNF induces slight soft tissue swelling. In any case, these results cannot be considered consistent or relevant. In terms of complications, we can report that the NNF caused only few adverse events. Patients mentioned after use headache, visible pressure marks, visible redness, and wrong impact on the nose. All these adverse events were mild and resolved under a temporary intermission or adaption of the NNF without the need for therapy.

There are various limitations to this study. First, the application protocol for the NNF used in this study was based on pilot experiences by the developers with only few subjects. Maybe another mode and duration of administration could have led to different and possibly more effective outcomes. Second, the compliance of the participants could barely be controlled or ensured which could have had an impact on the results. Third, there were many participants not completing the entire study, especially in the Cosmetic group, where high compliance was required, leading to decreased sample sizes over time. Possible reasons for this might have been high levels of frustration because the desired effect did not occur, discomfort wearing the NNF and the long duration of the course. Fourth, neither a placebo-device could be used to rule out a potential placebo effect caused by the NNF nor was there any pre-existing device for comparison. A final limitation might be a certain response bias in patient satisfaction, namely the tendency for patients to report in a way they feel is desirable. This can lead to overestimation of placebo effects of patient-reported outcomes (22). However, the power of impact is hardly assessable and can probably be neglected.

Even though the study design was chosen very carefully to limit potential biases to an absolute minimum and a most persona- lized device was used trying to create an optimized setting, we consider it to be almost impossible to entirely overcome the limitations mentioned above. Despite the optimal study settings with regular follow-up visits, the device has not proven to offer good results in an appropriate proportion of patients and we suspect it to perform even worse in a real-life setting.

\section{Conclusion}

Considering the results of this study, we conclude that even a perfectly customised external device to enhance rhinoplasty outcomes or to correct the shape of the nose without surgery does not seem to be effective enough and that further investigations in this field are not meaningful. Patients who are dissatisfied with their noses but are not willing to undergo surgery should consider other options.

\section{Acknowledgements}

This research was supported by the developers of the NNF who produced and provided the nose-formers at no charge. The authors like to thank Dr. med. Thomas Roth and Gianna Hartung for their helpful inputs as well as Nico Vogel and Marc Mosimann for their effort in early data analysis.

\section{Authorship contribution}

Conception and design of study and Acquisition of data: MBS; Analysis and interpretation of data and Drafting of the manuscript: MAD; Critical revision of the manuscript for important intellectual content: DH, MBS; Approval of the version of the manuscript to be published: MAD, DH, MBS.

\section{Conflict of interest}

None.

\section{References}

1. Andretto Amodeo C. The central role of the nose in the face and the psyche: review of the nose and the psyche. Aesthetic Plast Surg. 2007;31(4):406-10

2. American Society of Plastic Surgeons (ASPS): National Clearinghouse of Plastic Surgery Statistics 2016. 2017.

3. Moon HJ. Use of Fillers in Rhinoplasty. Clin Plast Surg. 2016:43(1):307-17.

4. Johnson ON, 3rd, Kontis TC. Nonsurgical Rhinoplasty. Facial Plast Surg. 2016;32 (5):500-6.

5. Jasin ME. Nonsurgical rhinoplasty using dermal fillers. Facial Plast Surg Clin North Am. 2013;21(2):241-52.

6. Turner $\mathrm{CH}$, Pavalko FM. Mechanotransduction and functional response of the skeleton to physical stress: the mechanisms and mechanics of bone adaptation. J Orthop Sci. 1998;3(6):346-55.
7. Duncan RL, Turner CH. Mechanotransduction and the functional response of bone to mechanical strain. Calcif Tissue Int 1995;57(5):344-58

8. Carvalho Trojan L, Andres Gonzalez-Torres L, Claudia Moreira Melo A, Barbosa de Las Casas E. Stresses and Strains Analysis Using Different Palatal Expander Appliances in Upper Jaw and Midpalatal Suture. Artificial organs. Artif Organs. 2017 Jun;41(6):E41-E51.

9. Krishnan V, Davidovitch Z. Cellular, molecular, and tissue-level reactions to orthodontic force. Am J Orthod Dentofacial Orthop. 2006;129(4):469 e1-32.

10. Melsen B. Tissue reaction to orthodontic tooth movement--a new paradigm. Eur J Orthod. 2001;23(6):671-81.

11. Strub B, Meuli-Simmen C, Bessler S. Photodocumentation in rhinoplasty surgery. Eur J Plastic Surg. 2013;36(3):141-8.

12. Jiang N, Guo W, Chen M et al. Periodontal
Ligament and Alveolar Bone in Health and Adaptation: Tooth Movement. Front Oral Biol. 2016;18:1-8.

13. Henneman S, Von den Hoff JW, Maltha JC Mechanobiology of tooth movement. Eur J Orthod. 2008;30(3):299-306.

14. Middleton J, Jones M, Wilson A. The role of the periodontal ligament in bone modeling: the initial development of a time-dependent finite element model. Am J Orthod Dentofacial Orthop. 1996;109(2):155-62.

15. Kaptchuk TJ, Miller FG. Placebo Effects in Medicine. N Engl J Med. 2015;373(1):8-9.

16. Hrobjartsson A, Gotzsche PC. Is the placebo powerless? An analysis of clinical trials comparing placebo with no treatment. N Engl J Med. 2001;344(21):1594-602.

17. Kaptchuk TJ, Stason WB, Davis RB et al. Sham device $v$ inert pill: randomised controlled trial of two placebo treatments. BMJ. 2006;332(7538):391-7 
18. Kaptchuk TJ, Goldman P, Stone DA, Stason WB. Do medical devices have enhanced placebo effects? J Clin Epidemiol. 2000;53(8):786-92.

19. Greenwald AG, Spangenberg ER Pratkanis AR, Eskenazi J. Double-Blind Tests of Subliminal Self-Help Audiotapes. Psychological Science. 1991;2(2):119-22.

20. Pavri S, Zhu VZ, Steinbacher DM Postoperative Edema Resolution following Rhinoplasty: A Three-Dimensional Morphometric Assessment. Plast Reconstr Surg. 2016;138(6):973e-9e.

21. Ozucer B, Yildirim YS, Veyseller B et al. Effect of Postrhinoplasty Taping on Postoperative Edema and Nasal Draping: A Randomized Clinical Trial. JAMA Facial Plast Surg.
2016;18(3):157-63.

22. Hrobjartsson A, Kaptchuk TJ, Miller FG. Placebo effect studies are susceptible to response bias and to other types of biases. Clin Epidemiol. 2011;64(11):1223-9.
PD Dr. med. Michael B. Soyka

Department of Otorhinolaryngology

Head and Neck Surgery

University Hospital Zurich

Frauenklinkstrasse 24

8091 Zurich

Switzerland

Tel: +41442551111

Fax: +414425545 56

E-mail: Michael.Soyka@usz.ch 